\title{
UFITA-SYMPOSIUM
}

\section{Aktuelle Rechtsfragen der deutschen und europäischen Filmförderung*}

\author{
Prof. Dr. Nadine Klass, LL.M. (Wellington) ${ }^{* *}$
}

Das Thema Filmförderung ist nicht neu. Im Gegenteil - die Wissenschaft und die Filmpraxis beschäftigt sich schon seit Jahrzehnten mit dieser Frage. ${ }^{1}$ Auch sind die verfassungsrechtlichen Grundfragen und Rahmenbedingungen der Filmförderung in Deutschland durch die Rechtsprechung des Bundesverfassungsgerichts sowie ein Bundesgesetz weitestgehend geklärt. ${ }^{2}$ Zentrale europarechtliche Fragen, wie die der beihilferechtlichen Vereinbarkeit nationaler Filmfördersysteme, wurden ebenfalls weitgehend beantwortet. ${ }^{3}$

Dennoch erscheint es sinnvoll, sich nochmals vertiefter mit dem Thema auseinanderzusetzen. ${ }^{4}$ Zum einen wirft die aktuell anstehende Umsetzung der revidierten Richtlinie über audiovisuelle Mediendienste ${ }^{5}$ neue Fragen auf: Zu klären ist beispielsweise, wie neue Akteure am Markt in die Filmförderung einzubinden sind. Zum anderen gerät aber auch die Territorialität als Kernmodell für die Filmfinanzierung, nicht nur in der Debatte um ein europäisches Urheberrecht, zunehmend unter Druck - auch das EU-Wettbewerbsrecht setzt der Gewährung territorialer Exklusivität strenge Grenzen. ${ }^{6}$ Die Angemessenheit des europäischen Filmfinanzierungssystems ist daher auch und gerade im Lichte der Herausforderungen der Digitalisierung, eines veränderten Marktes sowie eines neuen Zuschauerver-

* Einleitung zum Symposium „Aktuelle Rechtsfragen der deutschen und europäischen Filmförderung" des Instituts für Urheber- und Medienrecht (IUM), welches am 28.6.2019 in wissenschaftlicher Kooperation mit dem Institut für Europäisches Medienrecht (EMR) in München stattgefunden hat.

** Inhaberin des Lehrstuhls für Bürgerliches Recht, Recht des Geistigen Eigentums sowie deutsches und europäisches Verfahrensrecht an der Universität Mannheim.

1 Siehe beispielsweise: Dörr, ZUM 2010, 746; Castendyk, ZUM 2010, 757 sowie den Diskussionsbericht zum Symposium des IUM „Verfassungsrechtliche und kulturpolitische Grundsatzfragen der Filmförderung in Deutschland“ im Jahr 2010, ZUM 2010, 766.

2 Zur Vereinbarkeit der Filmabgabe mit dem GG vgl. BVerfG, NVwZ 2014, 646.

3 Vgl. insbesondere die Kinomitteilung 2013, ABl. C 332/1 vom 15.11.2013 und hierzu Lewke, K\&R 2012, 799; Werner, MMR 2014, 439.

4 Siehe auch zur aktuellen Diskussion: Martiny, ZUM 2018, 772; Gundel, ZUM 2019, 131.

5 Richtlinie (EU) 2018/1808 des Europäischen Parlaments und des Rates vom 14. November 2018 zur Änderung der Richtlinie 2010/13/EU zur Koordinierung bestimmter Rechts- und Verwaltungsvorschriften der Mitgliedstaaten über die Bereitstellung audiovisueller Mediendienste (Richtlinie über audiovisuelle Mediendienste) im Hinblick auf sich verändernde Marktgegebenheiten.

6 EuGH, Rs. 262/81, Slg. 1982, I-03381 Rn. 17 - Coditel II; EuGH ZUM 2011, 803 - Murphy; Commission Decision of 26.7.2016, Case AT.40023 - Cross-border access to pay-TV. 
haltens kritisch zu betrachten. Zudem ist vor diesem Hintergrund auch die Frage nach der Effektivität und möglichen Alternativmodellen erneut zu stellen. Ist eine territoriale Lizenzvergabe auf der Basis von Sprachfassungen sinnvoll? Welche Rolle spielen neue Filmfinanzierungsinstrumente wie Crowdfunding oder Produktplatzierung? Sollten die Filmauswertungsfenster abgeschafft oder flexibler ausgestaltet werden? ${ }^{7}$ Wie können audiovisuelle Mediendienste eingebunden werden?

Deutschland hat mit der Einbeziehung von Video-on-Demand-Anbietern schon eine Vorreiterrolle eingenommen ${ }^{8}$ - die geänderte Richtlinie ermöglicht es aber nunmehr allen Mitgliedstaaten, audiovisuelle Mediendienste in die Förderverpflichtung einzubeziehen und Verpflichtungen auch auf Anbieter auszudehnen, die aus dem Ausland "einstrahlen". Vorgesehen werden können beispielsweise Quotenregelungen mit Blick auf die Kataloge von Streaming-Anbietern, aber auch finanzielle Verpflichtungen wie die Einbeziehung in Filmförderfonds und andere Abgabensysteme. ${ }^{9}$

Fest steht jedenfalls, dass der europäische Film nach wie vor einen schweren Stand hat, denn während in Europa jährlich doppelt so viele Spielfilme produziert werden wie in den USA, beträgt der aktuelle Marktanteil der europäischen Filme in Europa typischerweise nur 20 bis $30 \% .^{10}$

$\mathrm{Zu}$ fragen ist daher auch, ob eine selektivere Vergabepolitik, etwa höherer Budgets für weniger Filme, einschließlich größerer Budgets beispielsweise für den Vertrieb oder die Werbung, die Chancen für EU-Filme erhöhen würde?

Nicht zuletzt wurden in den letzten Monaten einige wichtige Studien veröffentlicht, die sich - mit durchaus unterschiedlichem Fokus - mit der Frage der Filmförderung im europäischen Raum befassen. ${ }^{11}$

Dies allein war uns Anlass genug, dieses Thema nochmals in den Blick zu nehmen.

Ziel der Veranstaltung war es daher nicht nur, im Lichte dieser neuen rechtlichen und tatsächlichen Entwicklungen den rechtlichen status quo in Sachen Filmförderung zu analysieren, sondern auch kreativ in die Zukunft zu schauen und zu prüfen, welche rechtlichen

7 Vgl. hierzu Martiny, ZUM 2018, 772.

8 Vgl. § 153 Abs. 1, 2 Filmförderungsgesetz (FFG) vom 23.12.2016, BGBl. I S. 3413.

9 Vgl. Art. 13 AVMD-RL.

10 Siehe hierzu die Zahlen bei Poort/Hugenholtz/Lindhout/van Til, Research for CULT Committee Film Financing and the Digital Single Market: its Future, the Role of Territoriality and New Models of Financing, 2019.

11 Poort/Hugenholtz/Lindhout/van Til, Research for CULT Committee - Film Financing and the Digital Single Market: its Future, the Role of Territoriality and New Models of Financing, 2019; Cole, Guiding Principles in establishing the Guidelines for Implementation of Article 13 (6) AVMSD Criteria for exempting certain providers from obligations concerning European Works, 2019; Kanzler, Fiction film financing in Europe: A sample analysis of films released in 2016, 2018; Donders/Raats/Komorowski/Kostovska/Tintel/Iordache, Obligations on on-demand audiovisual media services providers to financially contribute to the production of European works. An analysis of European Member States' practices, 2018. 
Fragen sich künftig stellen und wie alternative Modelle der Filmförderung rechtlich ausgestaltet werden könnten.

Hierzu wurde in einem ersten Teil ein Blick auf die ökonomische Dimension und Relevanz der Förderung für die Filmproduktion geworfen und sowohl das tatsächliche Fördervolumen und deren Mittelverwendung als auch das Verhältnis zwischen europäischen und nationalen Fördersystemen analysiert. Sodann standen der deutsche und europäische Rechtsrahmen für die Filmförderung im Fokus, bevor in zwei Diskussionsrunden, zum einen mit dem Produzenten Max Wiedemann und zum anderen mit diversen Stakeholdern der Filmbranche, die aktuellen Herausforderungen der Filmförderung und eine mögliche Regulierung de lege ferenda diskutiert wurden. 